\title{
Neutron diffraction study of low-temperature magnetic phase diagram of an isosceles-triangular-lattice Ising antiferromagnet $\mathrm{CoNb}_{2} \mathrm{O}_{6}$
}

\author{
S. Kobayashi, ${ }^{1,}{ }^{*}$ S. Mitsuda, ${ }^{2}$ S. Hosaka, ${ }^{2}$ H. Tamatsukuri, ${ }^{2}$ T. Nakajima, ${ }^{2}$ H. Koorikawa, ${ }^{2}$ K. Prokeš, ${ }^{3}$ and K. Kiefer ${ }^{3}$ \\ ${ }^{1}$ Department of Materials Science and Engineering, Faculty of Engineering, \\ Iwate University, Ueda 4-3-5, Morioka 020-8551, Japan \\ ${ }^{2}$ Department of Physics, Faculty of Science, Tokyo University of Science, Shinjuku-ku, Tokyo 162-8601, Japan \\ ${ }^{3}$ Helmholtz-Zentrum Berlin für Materialien und Energie, Hahn-Meitner Platz 1, Berlin 14109, Germany
}

(Dated: June 10, 2016)

\begin{abstract}
Low-temperature magnetic phase diagram under magnetic fields along the orthorhombic $a$ axis for the isosceles-triangular-lattice antiferromagnet $\mathrm{CoNb}_{2} \mathrm{O}_{6}$ has been investigated by single-crystal neutron diffraction measurements down to $T=0.5 \mathrm{~K}$. The phase diagram consists of three magnetically ordered phases, i.e. antiferromagnetic (AF), incommensurate sinusoidal magnetic (IC), and saturated paramagnetic $(\mathrm{PM})$ phases, characterized by propagation wave vector, $\mathbf{Q}=\left(\begin{array}{lll}0 & \frac{1}{2} & 0\end{array}\right),\left(\begin{array}{l}0 \\ q\end{array}\right.$ $0)$, and $\left(\begin{array}{lll}0 & 0 & 0\end{array}\right)$, respectively. We found that a field-induced ferrimagnetic phase with $\mathbf{Q}=\left(\begin{array}{lll}0 & \frac{1}{4} & 0\end{array}\right)$ observed by previous neutron diffraction study down to $T=1.8 \mathrm{~K}[\mathrm{H}$. Weitzel et al., Phys. Rev. B 62, 12146 (2000)], does not exist as single equilibrium phase and always coexists with the other ordered phases near the triple point where the AF, IC, and saturated PM phases meet. We have also found that relaxation time of the system becomes extremely long below $T=0.6 \mathrm{~K}$, compared with time, which may be responsible for the appearance of magnetization plateaus at $T=0.5 \mathrm{~K}$. These plateaus have half the saturation magnetization, from which another field-induced state was inferred in previous magnetization measurements [T. Hanawa et al. J. Phys. Soc. Jpn. 63, 2706 (1994)].
\end{abstract}

PACS numbers: 75.25.-j, 75.30.Kz, 75.50.Ee, 75.60.Ej, 61.05.fm

\section{INTRODUCTION}

Antiferromagnets with geometrically frustrated triangular lattice have attracted considerable interest because of their diverse phase transitions and critical phenomena, originating from macroscopic degeneracy of the ground state $^{1,2}$. When is the triangular geometrical frustration partially released due to e.g. an orthorhombic distortion, the nearest-neighbor antiferromagnetic interaction of a triangular lattice is split into two inequivalent antiferromagnetic interactions, degeneracy of ground state is partially lifted and magnetic features reflecting ratio of antiferromagnetic interactions may appear. The investigation of a magnet with a frustrated isoscelestriangular lattice is of great importance, because such a system does not simply belong to the intermediate case between frustrated triangular-lattice antiferromagnets and unfrustrated magnets, but it also has a potential to exhibit unusual magnetic features absent in both magnets $^{3-6}$.

A columbite niobate, $\mathrm{CoNb}_{2} \mathrm{O}_{6}$, is one example of such isosceles-triangular-lattice antiferromagnets ${ }^{7-14}$. As shown in Figs. 1(a) and 1(b), magnetic $\mathrm{Co}^{2+}$ ions form quasi-one-dimensional (quasi-1D) ferromagnetic zigzag chains along the orthorhombic $c$ axis and form an isosceles-triangular lattice in the $a-b$ plane. In the zero field, the system exhibits successive magnetic phase transitions from the paramagnetic (PM) phase to the incommensurate sinusoidal magnetic (IC) phase with the temperature-dependent propagation wave vector, $\mathbf{Q}_{\mathrm{IC}}=$ $(0 q 0)$ at $T_{1} \sim 3.0 \mathrm{~K}$, and then to the noncollinear antiferromagnetic (AF) phase, with $\mathbf{Q}_{\mathrm{AF}}=\left(\begin{array}{l}0 \\ \frac{1}{2}\end{array}\right)$ at $T_{2} \sim 1.9$
$\mathrm{K}$, whose magnetic structure is shown in Fig. 1(b). $\mathrm{Co}^{2+}$ spins are confined to two different easy axes in the $a-c$ plane with a canting angle of $\theta_{0}\left(=31^{\circ}\right)$ from the $c$ axis, which originates from two crystallographically nonequivalent octahedral $\mathrm{CoO}_{6}$ sites. Extensive investigations for magnetic fields along the $c$ axis revealed various magnetic features reflecting spin frustration on the isosceles triangular lattice, such as magnetic-structure-dependent spin correlations along the $a$ axis ${ }^{8}$, anisotropic and extremely slow domain-growth kinetics ${ }^{9,11}$ and a rich magneticfield $(H)$-vs-temperature $(T)$ phase diagram which consists of AF, IC, and saturated PM phases, field-induced ferrimagnetic (FR) phase with $\mathbf{Q}=\left(\begin{array}{lll}0 & \frac{1}{3} & 0\end{array}\right)$, higherfield ferrimagnetic (HHFR) phase with $\mathbf{Q}=\left(\begin{array}{lll}0 & \frac{1}{2} & 0\end{array}\right)$, and low-temperature higher-field ferrimagnetic (LHFR) phase with $\mathbf{Q}=\left(0 \frac{1}{3} 0\right)^{10,13-15}$. These features have been qualitatively explained within an isosceles-triangular lattice Ising model with nearest-neighbor antiferromagnetic interaction $J_{1}$ and next one $J_{2}$, with $\gamma=J_{1} / J_{2}$ being 1.33, not far from the 1.0 of the triangular lattice ${ }^{16}$.

When magnetic field is applied along the direction in the $a-c$ plane at a finite angle to the $c$ axis, interesting magnetic features are expected because of the two different easy axes. Weitzel et al. performed neutron diffraction measurements down to $T=1.7 \mathrm{~K}$ under magnetic fields along various directions in the $a-c$ plane and proposed a $H-T$ magnetic phase diagram including several field-induced ferrimagnetic phases with different propagation vectors, whose stability depends on the angle of magnetic field to the $c$ axis $^{14}$. While for angles up to $\sim 45^{\circ}$, magnetic phases constituting the $H-T$ phase diagram are the same as those for field along the $c$ axis, 


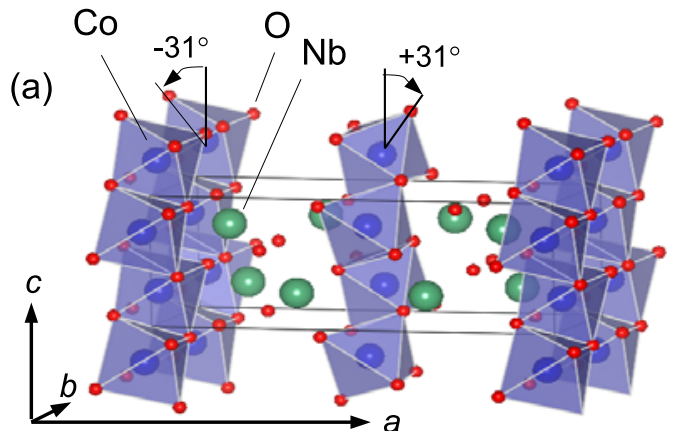

(b) AF

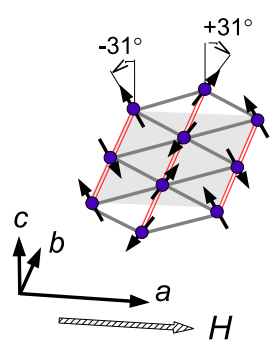

(c) FR2

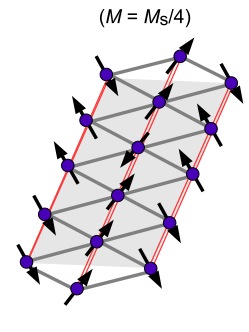

(d) Saturated PM $\left(M=M_{\mathrm{s}}\right)$

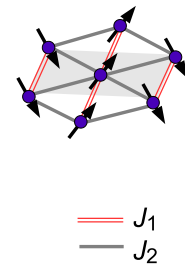

FIG. 1. (Color online) (a) Crystal structure of $\mathrm{CoNb}_{2} \mathrm{O}_{6}$. Two different easy axes exist in the $a-c$ plane with a canting angle of $\pm 31^{\circ}$ from the $c$ axis, which originate from the two different octahedral $\mathrm{CoO}_{6}$ sites. Magnetic structure in (b) AF, (c) FR2, and (d) saturated PM phases under applied fields along the $a$ axis. Magnetic $\mathrm{Co}^{2+}$ ions form an isosceles-triangular lattice in the $a-b$ plane with interchain antiferromagnetic exchange interactions, $J_{1}$ and $J_{2}$. The arrows represent $\mathrm{Co}^{2+}$ spins. All spins are confined on easy axes on the $a-c$ plane canted from the $c$ axis. The shaded area corresponds to a magnetic unit cell.

another ferrimagnetic phase with $\mathbf{Q}=\left(\begin{array}{lll}0 & \frac{1}{4} & 0\end{array}\right)$ shows up between the two FR and HHFR phases for angles greater than $\sim 45^{\circ}$; hereafter we call this ferrimagnetic phase FR2 one. With increasing the angle to $\sim 60^{\circ}$, the FR phase completely disappears and for applied fields along the $a$ axis only the FR2 phase shows up as a fieldinduced ferrimagnetic phase. These magnetic phase diagrams were qualitatively reproduced by mean-field numerical approach similar to that by Boehm et al. ${ }^{17}$, assuming a full Hamiltonian with competing exchange interactions on an isosceles triangular lattice, crystal field, dipolar-dipolar interactions etc ${ }^{14}$.

In spite of a deeper understanding for magnetic features under applied fields in this system ${ }^{7,8,10,13,14}$, two unresolved issues remain for applied fields along the $a$ axis.

(1) From neutron diffraction measurements down to $T$ $=1.7 \mathrm{~K}$, Weitzel et al. proposed a $H_{/ / a}-T$ magnetic phase diagram that consists of the AF, IC, FR2, and saturated PM phases, where the FR2 phase appears in the intermediate magnetic-field and temperature ranges of roughly $H=700-1200$ Oe and $T=1.7-2.5 \mathrm{~K}^{14,18}$. On the basis of magnetic reflections obtained under applied fields forming an angle of $\sim 45^{\circ}$ to the $c$ axis, Weitzel et al. proposed a magnetic structure of the FR2 and saturated PM phases as shown in Figs. 1(c) and 1(d), respectively. Although the proposed FR2 structure has a saturation magnetization of $M_{\mathrm{s}} / 4$ along the $a$ axis, magnetization along the $a$ axis monotonically increases with field in the intermediate magnetic-field range and a magnetization plateau corresponding to the appearance of the FR2 phase is not observed ${ }^{7,14}$. This implies that the FR2 state is metastable and does not exist as a thermodynamically equilibrium phase for applied fields along the $a$ axis. Actually, there is a large discrepancy with the calculated $H_{/ / a}-T$ phase diagram assuming the full Hamiltonian, obtained by Weitzel et al.; a phase transition from the AF to the saturated PM phase occurs directly, and the field-induced FR2 phase does not appear in the calculated $H_{/ / a}-T$ phase diagram.

(2) Hanawa et al. surveyed a $H_{/ / a}-T$ magnetic phase diagram by magnetization and specific-heat measurements down to $T=0.5 \mathrm{~K}^{7}$. They have found that a saturated PM phase is field induced under low fields of $\sim 1.5 \mathrm{kOe}$ at all measured temperatures below $T_{1}$. In particular, at the lowest measured temperature of 0.5 $\mathrm{K}$, the magnetization plateau with half saturation magnetization, $M_{\mathrm{s}} / 2$, is seen, implying an intermediate fieldinduced phase between the AF and saturated PM phases. The details of this intermediate phase inferred by Hanawa et al. and its relation with the higher temperature FR2 phase observed by Weitzel et al. remain unclear.

In this study, we have performed low-temperature neutron diffraction and magnetization measurements down to $T=0.5 \mathrm{~K}$ on a single crystal of $\mathrm{CoNb}_{2} \mathrm{O}_{6}$ under a magnetic field applied along the $a$ axis, in order to investigate how a field-induced ferrimagnetic phase characterized by $\mathbf{Q}_{\mathrm{FR} 2}=\left(\begin{array}{lll}0 & \frac{1}{4} & 0\end{array}\right)$, suggested by Weitzel et al. is extended to lower temperature region in the $H_{/ / a}-T$ phase diagram and how it connects with $1 / 2$ magnetization plateau at $T$ $=0.5 \mathrm{~K}$ reported by Hanawa etal. The low-temperature $\mathrm{H}_{/ / \mathrm{a}}-\mathrm{T}$ magnetic phase diagram of $\mathrm{CoNb}_{2} \mathrm{O}_{6}$ has also been constructed.

\section{EXPERIMENT}

A single crystal with dimensions $5 \times 5 \times 10 \mathrm{~mm}^{3}$, grown with the flux-growth technique ${ }^{19}$ and used in previous neutron diffraction study ${ }^{8,10}$, was used. Neutron diffraction measurements were performed using the twoaxis diffractometer, E4, installed in Helmholtz-Zentrum Berlin. A pyrolytic-graphite filter was used to eliminate second-order contamination, and incident neutrons with a wavelength of $2.44 \AA$ were used. A vertical external field along the $a$ axis up to $2 \mathrm{kOe}$ and temperature down to $0.5 \mathrm{~K}$ were provided by a $5 \mathrm{~T}$ superconducting cryomagnet (VM-3) with a Helium-3 insert. The sample was mounted so as to access the $(0 k l)$ scattering plane in the reciprocal lattice.

The dc magnetic measurements down to $T=0.5$ 
$\mathrm{K}$ were performed using a commercial superconducting quantum interference device (SQUID) magnetometer (Quantum Design MPMS-XL) equipped with a Helium-3 refrigerator (iHelium3). A magnetic field along the $a$ axis up to $2 \mathrm{kOe}$ was applied and the magnetization along the $a$ axis was measured as a function of magnetic field in the hysteresis mode.

\section{RESULTS AND DISCUSSION}

\section{A. Neutron diffraction measurements}

Figures $2(\mathrm{a})-2(\mathrm{~d})$ show the $\left(\begin{array}{lll}0 & k & 0\end{array}\right)$ reciprocal-lattice scans, taken with increasing magnetic field at several temperatures below $T_{1}$. At the lowest temperature of $0.5 \mathrm{~K}$, a AF peak with $q=\frac{1}{2}$ appears in zero field and starts to diminish at around $H=500$ Oe with increasing magnetic field, associated with the appearance of the $(0$ $10)$ peak of the saturated PM phase as shown in Fig. 2(a). As the magnetic field further increases, the PM peak develops and the field-induced AF-PM phase transition takes place at around $H=700$ Oe. As shown in the inset in Fig. 2(a), the AF peak persists up to a high magnetic field of $1.5 \mathrm{kOe}$ and no field-induced intermediate phase with $q$ different from 0 and $\frac{1}{2}$, was observed between the AF and PM phases.

At $T=1.85 \mathrm{~K}$, a AF peak appears in zero field and a field-induced $\mathrm{AF}-\mathrm{IC}$ phase transition occurs at a low applied field of $\sim 150$ Oe, with the coexistence of both $\mathrm{AF}$ and IC peaks as shown in Fig. 2(b). As the magnetic field increases, IC peaks located at $(0 q 0)$ and $\left(\begin{array}{lll}0 & 1-q & 0\end{array}\right)$ with $q \sim 0.48$ gradually shift toward the $\left(\begin{array}{lll}0 & 0 & 0\end{array}\right)$ and $\left(\begin{array}{ll}0 \\ 0\end{array}\right.$ $10)$ PM peak positions, respectively. At the same time, IC peaks become strongly broadened and their peak intensities largely decrease, associated with the evolution of the ( $\left.\begin{array}{lll}0 & 1 & 0\end{array}\right)$ PM peak. At around $H=800$ Oe, a broad magnetic peak centered at $k \sim 0.75$ was observed beside a broad IC peak at $k \sim 0.7$ as shown in the inset in Fig. 2 (b). Since the width of the magnetic peak at $k \sim 0.75$ is comparable to that of the IC peak, the magnetic peak at $k \sim 0.75$ may be composed of many magnetic peaks with different propagation wave numbers, which are equal and close to $q=\frac{1}{4}$ of the FR2 state. Hereafter, we call this broad magnetic peak centered at around the FR2 peak position FR2 peak for simplicity. With increasing magnetic field, the intensity of both IC and FR2 peaks diminish and the IC peak disappears around $H=1000$ Oe, although the broad FR2 peak persists up to slightly higher field of 1100 Oe above which the saturated PM ordering is almost achieved. Such similar field dependence of a FR2 peak was observed at $T=2.2 \mathrm{~K}$ just above $T_{2}$ as shown in Fig. 2(c), where IC ordering is stabilized in zero field. On the other hand, at high temperatures above $\sim 2.5 \mathrm{~K}$, a simple field-induced IC-PM phase transition takes place with increasing magnetic field and no FR2 peak was observed as shown in Fig. 2(d). These observations strongly indicate that the FR2 state with $q$
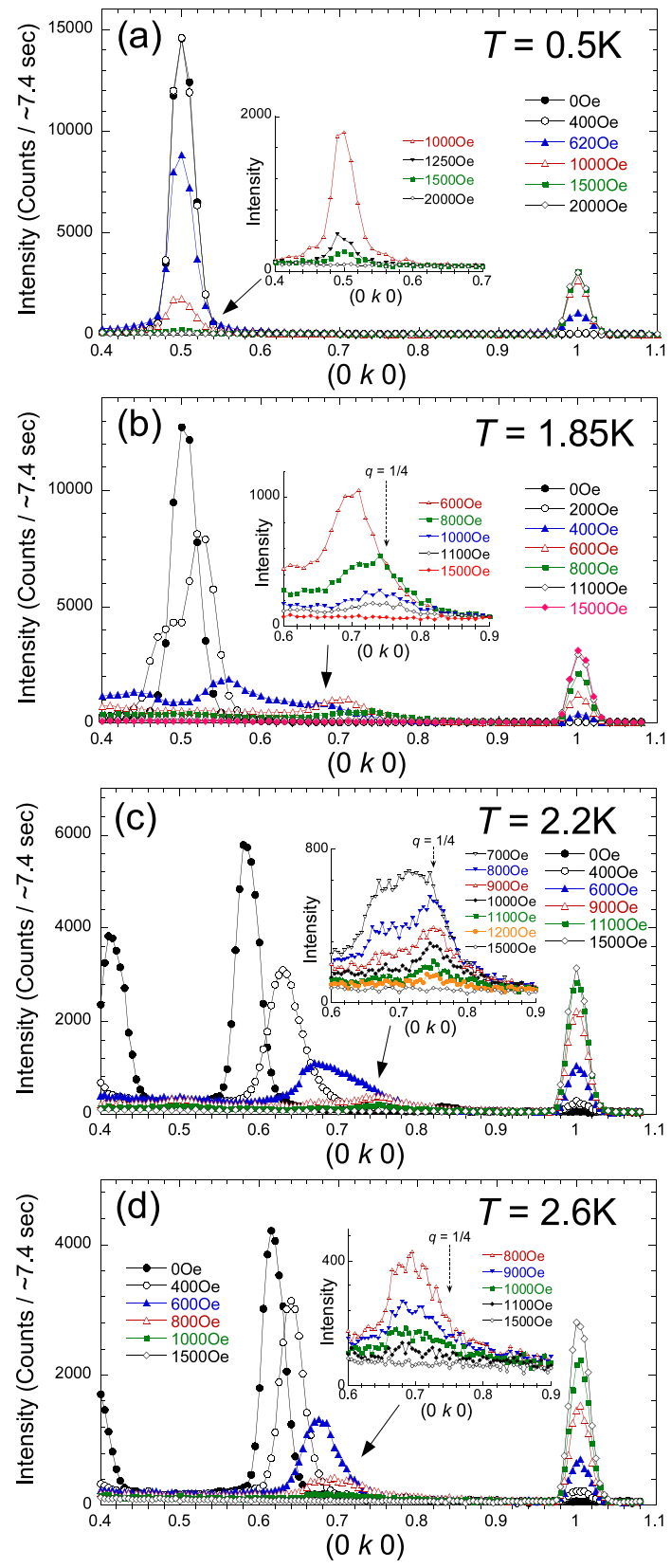

FIG. 2. (Color online) Magnetic-field dependence of the representative neutron diffraction $(0 k 0)$ reciprocal-lattice scans at (a) $T=0.5 \mathrm{~K}$, (b) $T=1.85 \mathrm{~K}$, (c) $T=2.2 \mathrm{~K}$, and (d) $T$ $=2.6 \mathrm{~K}$. The insets show their enlargement in the medium field range.

$=\frac{1}{4}$, observed by Weitzel et al., does not appear as single equilibrium phase and always coexists with IC and/or saturated PM phases.

In Figs. 3(a)-3(h), the magnetic-field dependence of $q$ and integrated intensities, obtained from these $(0 k 0)$ scans, are summarized. For magnetic fields where both IC and FR2 peaks overlap, data were obtained by leastsquares fits assuming double Gaussian profiles. Note 


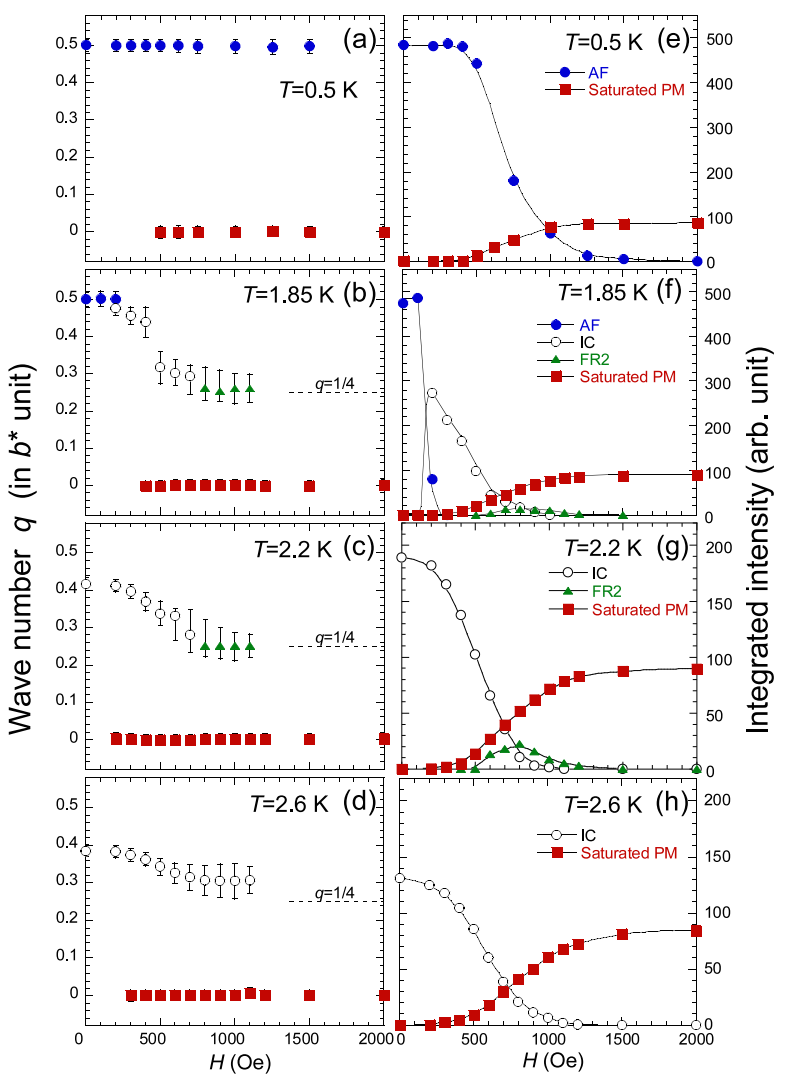

FIG. 3. (Color online) Magnetic-field dependence of the propagation wave number, $q$, in the $b^{*}$ direction $[(\mathrm{a})-(\mathrm{d})]$ at several temperatures below $T_{1}$. The closed circles, open circles, triangles, and squares represent data of AF, IC, FR2, and saturated PM peaks, respectively. For magnetic fields at $T$ $=1.85$ and $2.2 \mathrm{~K}$, where both IC and FR2 peaks coexist, $q$ was taken from the peak position. To show how IC and FR2 peaks are anomalously broadened, the full width at the half maximum of a magnetic peak is given as the vertical bars in (a) -(d). Magnetic-field dependence of integrated intensities of the $\left(\begin{array}{lll}0 & 1-q & 0\end{array}\right)$ peak $[(\mathrm{e})-(\mathrm{h})]$ at several temperatures below $T_{1}$. The solid lines through the data are guides to eyes.

again that data for the FR2 peak may originate from many magnetic peaks with different $q$ equal and close to $\frac{1}{4}$. As shown in Figs. 3(f) and $3(\mathrm{~g})$, the integrated intensity of the FR2 peak maximizes in the intermediate magnetic fields of 800-900 Oe and temperatures of $2.2 \mathrm{~K}$, but the intensity of the FR2 peak is much smaller than that of the IC and/or PM peaks.

Next, in order to investigate how the FR2 peak extends into the low-temperature region, we performed $(0$ $k 0$ ) reciprocal-lattice scans at fixed magnetic field of 900 Oe, on cooling from $T=2.2 \mathrm{~K}$. As shown in Fig. 4, at $H$ $=900$ Oe and $T=2.2 \mathrm{~K}$, several broad magnetic peaks were observed in addition to the sharp ( $\left.\begin{array}{lll}0 & 1 & 0\end{array}\right)$ PM peak; a (0 1- $q 0)$ FR2 peak with $q=\frac{1}{4}$ located at $k=\frac{3}{4}$, its $2 q$ and $1-2 q$ one at $k=\frac{1}{2}$, and a small (0 1-q 0$)$ IC peak with $q \sim 0.32$ at $k \sim 0.68$. As the temperature decreases, the

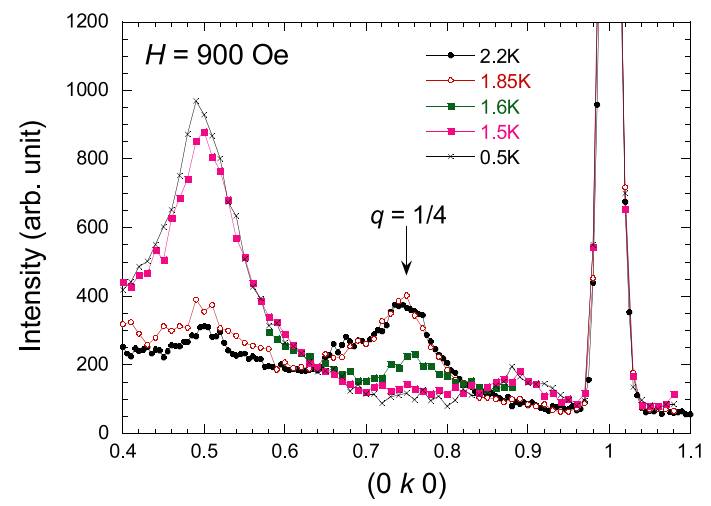

FIG. 4. (Color online) Temperature dependence of the neutron diffraction profile of the $(0 k 0)$ scans, taken at $H=900$ Oe.

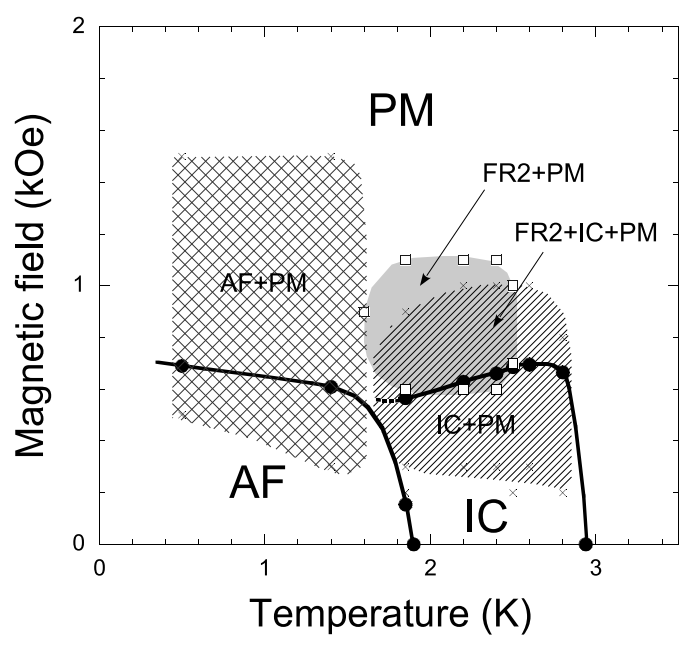

FIG. 5. The $H-T$ phase diagram for applied field along the $a$ axis. The solid circles at a finite field represent critical fields, determined from neutron integrated intensities, whereas those in zero field are taken from Ref. 8. The cross-hatched and hatched areas represent the $H-T$ region, where the AF and IC phases coexist with the saturated PM one, respectively. The boundaries of such coexistence regions, were determined by using the data points shown by the crosses. The $H-T$ region where the FR2 peak was observed, was shown by the shaded area, surrounded by square symbols. Therefore, the shaded area means the coexistence region for the FR2 and PM states, while the region that the shaded and hatched ones overlap means the coexistence region for the FR2, IC, and saturated PM states.

FR2 peak at $k=\frac{3}{4}$ and the IC one suddenly start to diminish just below $T=1.85 \mathrm{~K}$ and completely disappear at $T=1.5 \mathrm{~K}$. This accompanies a large increase of the $(0$ $\left.\frac{1}{2} 0\right)$ peak intensity as well as a slight decrease of the (0 1 0 ) one. These observations suggest that most of magnetic domains, which contribute to broad FR2 and IC peaks are changed into AF ordered regions at low temperatures 

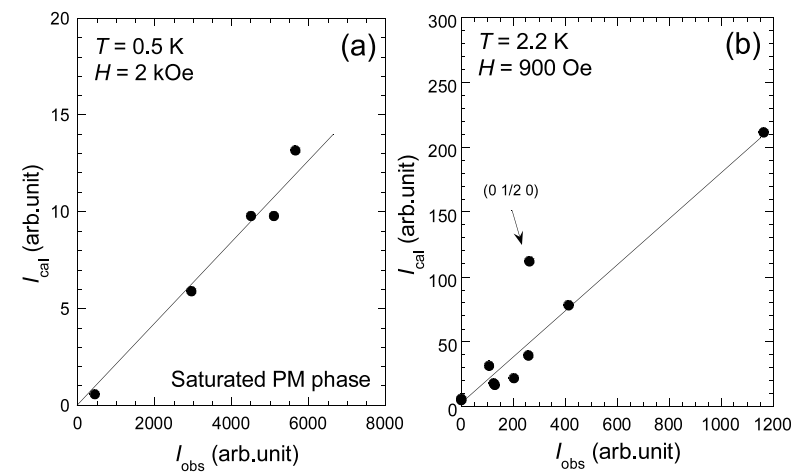

FIG. 6. (Color online) The observed and calculated integrated intensities, taken at (a) $H=2 \mathrm{kOe}$ and $T=0.5 \mathrm{~K}$ for the saturated PM phase, and (b) $H=900 \mathrm{Oe}$ and $T=2.2 \mathrm{~K}$ for a coexistence phase of the saturated PM and FR2 phases.

below $T \sim 1.5 \mathrm{~K}$. Note that below $T \sim 1.5 \mathrm{~K}$ a small and broad magnetic peak around $k=0.9$ as well as strong broadening of the AF peak were observed; this may reflect a difficulty of magnetic moment rearrangement at low temperatures to form a long-range AF order.

In Fig. 5, the $H-T$ magnetic phase diagram obtained from the present neutron diffraction study is summarized. A critical field was defined as a midpoint between magnetic fields for higher and lower-field phases at which their integrated intensities become a half their maximum value. The phase diagram consists of three magnetic phases of the AF, IC, and saturated PM phases. Coexistence regions for the $\mathrm{AF}$ and $\mathrm{PM}$ orders and for the IC and PM orders extend over a wide $H-T$ area, i.e., approximately $1 \mathrm{kOe}$ along the field axis. In addition, the FR2 state, which may be assembly of magnetic states with different wave numbers, which are equal or close to $\frac{1}{4}$, always coexists with other ordered state and appears near the triple point where the AF, IC, and saturated PM phases meet. This may reflect the presence of many quasi-degenerate ground states with $q$ from 0 to $\frac{1}{2}$ near the triple point. Note that in the $H-T$ magnetic phase diagram determined by Weitzel et al. ${ }^{14}$, the FR2 phase is represented as a stable magnetic phase, although the detail how the phase boundary of the FR2 phase was determined, is not clear. Nevertheless, we can not rule out a possibility of a sample dependence of the volume fraction of the FR2 state due to e.g. sample imperfection that stabilizes the FR2 state.

Next, in order to investigate detailed magnetic structures under magnetic fields along the $a$ axis, we surveyed integrated intensities of several magnetic Bragg points for both saturated PM and FR2 states. The magnetic structural analysis was carried out because the proposed magnetic structures of Figs. 1(c) and 1(d) were based on neutron diffraction measurements for applied fields forming an angle of $\sim 45^{\circ}$ to the $c$ axis, and not for applied fields along the $a$ axis. First, we show the results for

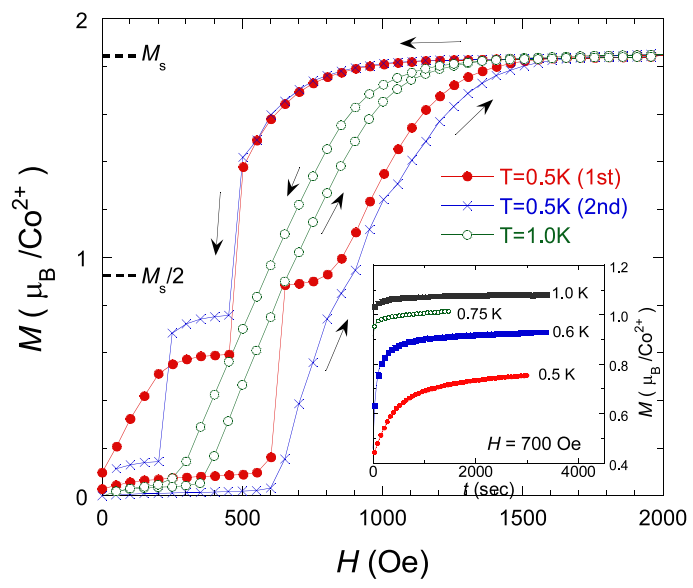

FIG. 7. (Color online) (a) Magnetization as a function of magnetic field along the $a$ axis, taken at $T=0.5 \mathrm{~K}$. The 1 st and 2 nd trials were independently performed with the same experimental setup. For comparison, the data taken at $T=$ $1.0 \mathrm{~K}$ is also given. The inset shows the time dependence of magnetization at $H=700$ Oe after field quench from zero field at several temperatures at and below $T=1.0 \mathrm{~K}$.

the saturated PM phase. Integrated intensities were collected by $\theta-2 \theta$ scan for five magnetic Bragg points in $(0 k l)$ zone, where contamination due to nuclear Bragg peak can be ignored. Figure 6(a) shows a relation of observed integrated intensities, $I_{\mathrm{obs}}$, with calculated ones, $I_{\text {obs }}$, assuming a magnetic structure of Fig. 1(d). The good linear relationship confirms a magnetic structure of Fig. 1(d) in the saturated PM phase, being consistent with previous studies ${ }^{7,13,14}$.

Next, we pay attention to a magnetic structure of the FR2 state. Figure $6(\mathrm{~b})$ shows a $I_{\text {obs }}$ versus $I_{\text {cal }}$ curve, assuming a FR2 structure of Fig. 1(c). Here, integrated intensities for magnetic Bragg peaks at $(0 k 0)$ and $(0 k$ 1 ) with $k$ up to 2.25 , expected from the FR2 structure with $\mathbf{Q}_{\mathrm{FR} 2}=\left(\begin{array}{lll}0 & \frac{1}{4} & 0\end{array}\right)$ were collected at $H=900$ Oe and $T=2.2 \mathrm{~K}$. Intensities for $k=$ integer were eliminated because of a significant contribution of the saturated PM phase. One can see that there is a rough proportional relationship between $I_{\text {obs }}$ and $I_{\text {cal }}$, but data for the $\left(0 \frac{1}{2}\right.$ $0)$ reflection exhibits a large deviation and the detailed magnetic structure was not identified. We have tested also other magnetic structural models with magnetic unit cell along the $b$ axis of four chemical ones, which are energetically probable within our isosceles-triangular-lattice Ising model ${ }^{8}$, but such a proportionality could not be improved. The observed poor proportionality would be attributed to the presence of many magnetic domains characterized by different $q$, which yields a strong broadening of a FR2 peak. 


\section{B. Magnetic measurements}

Previous magnetization measurements by Hanawa et al. reported the appearance of magnetization plateaus with $M \sim M_{\mathrm{s}} / 2$ at $T=0.5 \mathrm{~K}$ in the intermediate field range between the $\mathrm{AF}$ and the saturated $\mathrm{PM}$ phases ${ }^{7}$. This indicates the appearance of another field-induced intermediate magnetic state. However, in the present neutron diffraction study, the corresponding magnetic state was not confirmed at $T=0.5 \mathrm{~K}$. In order to elucidate the reason for the appearance of such $1 / 2$ magnetization plateaus, we carried out the detailed dc magnetization measurements with a commercial SQUID magnetometer. Note that Hanawa et al. measured $M-H$ curves using an induction method with continuously sweeping magnetic field with a rate of $\sim 67 \mathrm{Oe} / \mathrm{s}$, whereas a magnetic field was varied step-by-step with an interval of $50 \mathrm{Oe}$ with a similar rate of $\sim 40 \mathrm{Oe} / \mathrm{s}$ in the present dc measurements. Figure 7 shows magnetization as a function of magnetic field along the $a$ axis, taken at $T=0.5 \mathrm{~K}$. The $M-H$ curves were measured with a field cycle of $0 \rightarrow 2000 \rightarrow 0$ Oe. Two independent measurements, i.e. 1 st and 2 nd trials, were done to see the reproducibility of data. As the magnetic field increases, a sudden increase of magnetization occurs at $H \sim 600$ Oe for the 1st trial, whereas for the 2nd trial, a clear magnetization jump was not observed. On the descending branch of $M-H$ curves, a magnetization jump occurs at $H \sim$ 500 Oe for both cases, but, another magnetization jump occurs at $H \sim 200$ Oe for the 2 nd trial. Just after the magnetization jump, the magnetization reaches almost the same value as that at $T=1.0 \mathrm{~K}$, and then shows magnetization plateaus; $M \sim 0.5 M_{\mathrm{s}}$ for increasing field and $0.44 M_{\mathrm{s}}, 0.33 M_{\mathrm{s}}$ for decreasing field. These observations indicate that magnetization plateaus implying an intermediate state at $T=0.5 \mathrm{~K}$ are not reproducible.

As revealed in our previous neutron diffraction study for applied fields along the $c$ axis ${ }^{10}$, relaxation time of this system is extremely long at low temperatures below $\sim 0.6 \mathrm{~K}$, compared with our observation time. Therefore, it is possible that energetically unfavorable ferromagnetic quasi-1D chains along the $c$ axis are frozen, leading to the appearance of magnetization plateaus with various magnetization values. In order to check a relaxation behavior for applied fields along the $a$ axis, we have measured the time dependence of magnetization at $H=700$ Oe after a sudden field change from $H=0$ Oe at several temperatures between 0.5 and $1.0 \mathrm{~K}$ as shown in the inset in Fig. 7. At $T=1.0 \mathrm{~K}$, the magnetization just after reaching $H$ $=700$ Oe already has a high value, which is almost the same as that of Fig. 7, and is weakly dependent on time, indicating that the system goes into an equilibrium state with short observation time. The magnetization just after field change becomes lower with decreasing temperature and exhibits extremely long time relaxation below $T$ $=0.6 \mathrm{~K}$. Even after $3 \mathrm{ksec}$, the magnetization at $T=0.5$ $\mathrm{K}$ is approximately $70 \%$ of the value at $T=1.0 \mathrm{~K}$. These results suggest that relaxation time becomes extremely long below $T \sim 0.6 \mathrm{~K}$ also for applied fields along the $a$ axis.

At $T=0.5 \mathrm{~K}$, the magnetization jump frequently occurs as the magnetic field increases or decreases, and it accompanies a reversal of many ferromagnetic quasi-1D chains running along the $c$ axis. Just after the reversal the magnetization reaches nearly the same value as that at $T=1.0 \mathrm{~K}$. Therefore, the system would be in a nearly equilibrium state at a given magnetic field just after the magnetization jump. However, the system may not follow a subsequent change of external field due to extremely long time relaxation, being responsible for the appearance of the magnetization plateaus at $T=0.5 \mathrm{~K}$ shown in Fig. 7. Note that although a magnetization increase accompanies an increase in the PM peak intensity, a sudden change of the PM peak intensity due to a magnetization jump, was not observed in the magnetic field dependence of the integrated intensity at $T=0.5$ $\mathrm{K}$ shown in Fig. 3(e); this may be due to the fact that a large magnetization jump did not occur as in the case of the 2nd trial shown in Fig. 7 when the neutron data of Fig. 3(e) were collected with increasing field, or due to the poor number of neutron data points in Fig. 3(e) compared with the magnetization data.

\section{SUMMARY}

Low-temperature magnetic phase diagram under magnetic field along the $a$ axis in an isosceles-triangularlattice antiferromagnet $\mathrm{CoNb}_{2} \mathrm{O}_{6}$ has been investigated with neutron diffraction measurements down to $T=0.5$ $\mathrm{K}$. We found that the field-induced FR2 phase characterized by the propagation wave vector $\mathbf{Q}_{\mathrm{FR} 2}=\left(\begin{array}{ll}0 & \frac{1}{4} \\ 0\end{array}\right)$ does not exist as a thermodynamically equilibrium phase. In the $H-T$ phase diagram, only AF, IC and saturated PM phases appear, and the FR2 state always appears as a coexistence state with other ordered phases in an intermediate temperature and magnetic field near the triple point where the AF, IC and saturated PM phases meet. From dc magnetization measurements down to $T=0.5$ $\mathrm{K}$, the field-induced intermediate state with half the saturation magnetization, inferred from the magnetization plateaus at $T=0.5 \mathrm{~K}$, was found to originate from longtime relaxation of the system, which becomes extremely slow below $T \sim 0.6 \mathrm{~K}$.

\section{ACKNOWLEDGMENT}

This work was supported by a Grant-in-Aid for Scientific Research (C) (Grant No. 23540424 and 26400369) from JSPS, Japan. We thank HZB for the allocation of neutron beamtime. The neutron diffraction experiments at HZB was carried out according to Proposal No. PHY01-3176. The crystal structure figure was drawn using the VESTA software ${ }^{20}$. 
* koba@iwate-u.ac.jp

${ }^{1}$ H.T. Diep, Frustrated Spin Systems (World Scientific, Singapore, 2004).

2 M.F. Collins and O.A. Petrenko, Can. J. Phys. 75, 605 (1997).

3 J. Stephenson, J. Math. Phys. 11, 420 (1970).

4 J. Stephenson, Can. J. Phys. 48, 2118 (1970).

5 J. Doczi-Reger and P.C. Hemmer, Physica 108A, 531 (1981).

${ }^{6}$ S.B. Lee, R.K. Kaul, and L. Balents, Nature Phys. 6, 702 (2010).

7 T. Hanawa, K. Shinkawa, M. Ishikawa, K. Miyatani, K. Saito, and K. Kohn, J. Phys. Soc. Jpn. 63, 2706 (1994).

8 S. Kobayashi, S. Mitsuda, M. Ishikawa, K. Miyatani, and K. Kohn, Phys. Rev. B 60, 3331 (1999).

${ }^{9}$ S. Kobayashi, S. Mitsuda, T. Jogetsu, J. Miyamoto, H. Katagiri, and K. Kohn, Phys. Rev. B 60, R9908 (1999).

10 S. Kobayashi, S. Mitsuda, and K. Prokes, Phys. Rev. B 63, 024415 (2000).

11 S. Kobayashi, H. Okano, T. Jogetsu, J. Miyamoto, and S. Mitsuda, Phys. Rev. B 69, 144430 (2004).

12 S. Kobayashi, S. Hosaka, H. Tamatsukuri, T. Nakajima, S. Mitsuda, K. Prokes, and K. Kiefer, Phys. Rev. B 90,
060412(R) (2014).

13 C. Heid, H. Weitzel, P. Burlet, M. Bonnet, W. Gonschorek, T. Vogt, J. Norwig, and H. Fuess, J. Mag. Mag. Mater. 151, 123 (1995).

${ }^{14}$ H. Weitzel, H. Ehrenberg, C. Heid, H. Fuess, and P. Burlet, Phys. Rev. B 62, 12146 (2000).

15 The FR and HHFR phases in Ref. 10 correspond to the SF1 and SF2 phases given in Ref. 14, respectively. The LHFR phase appears only at low temperatures below $T \sim$ $0.6 \mathrm{~K}$ (Ref. 10) and was not reported in neutron diffraction study down to $T=1.5 \mathrm{~K}$ of Ref. 14

${ }^{16}$ In the present study, we redefined the $J_{2} \cos 2 \theta_{0}$ of our previous study (Ref. 8) as $J_{2}$ for simplicity. Here, the term $\cos 2 \theta_{0}$ originates from the two different easy axes along which the Co spins align.

17 J. von Boehm and P. Bak, Phys. Rev. Lett. 42, 122 (1979).

18 The FR2 and saturated PM phases correspond to the SF3 and $F_{a}$ phases given in Ref. 14, respectively.

19 B.M. Wanklyn, B.J. Garrard, and G. Garton, Mater. Res. Bull. 11, 1497 (1976).

${ }^{20}$ K. Momma and F. Izumi, J. Appl. Crystallogr. 44, 1272 (2011). 